\title{
A Study on the Development of Organic Thin Film Solar Cell Device With Optimized Hole Transfer Layer of PEDOT:PSS
}

\author{
Paik-Kyun Shin ${ }^{1}$, Kumar Palanisamy ${ }^{2}$, Abhirami Kumar ${ }^{2}$, Katsuhiko Kato $^{2} \&$ Shizuyasu Ochiai $^{2}$ \\ ${ }^{1}$ Department of Electrical Engineering, Inha University, Incheon, South Korea \\ ${ }^{2}$ Department of Electrical Engineering, Aichi Institute of Technology, Toyota, Japan \\ Correspondence: Shizuyasu Ochiai, Department of Electrical Engineering, Aichi Institute of Technology, Toyota, \\ Aichi 470-0395, Japan. Tel: 81-565-48-8121. E-mail: ochiai@aitech.ac.jp
}

Received: October 8, 2013 Accepted: October 22, 2013 Online Published: November 29, 2013

doi:10.5539/jmsr.v3n1p57 URL: http://dx.doi.org/10.5539/jmsr.v3n1p57

\begin{abstract}
Organic thin film solar cells show generally lower power conversion efficiencies than those of the solar cells based on inorganic active materials. To solve the problem diverse research works have been tried: development of new organic semiconductors; modification of internal structure of active layers by means of organic solvent; design and fabrication of vertical device structure; introduction of buffer layer on the side of cathode electrode. Nevertheless, the buffer layer on the side of anode has not been fully optimized. This study is focused on the representative anode-side-buffer layer of Poly(3,4-ethylenedioxythiophene)(PEDOT): poly(styrenesulfonate) (PSS). PEDOT:PSS is an excellent conducting polymer material with favorably high optical transparency for almost entire range of visible wavelength, which is widely used for organic thin film solar cells as well as for other organic material based electronic devices such as organic light emitting diodes. Aiming for further performance enhancement of organic thin film solar cell device, an optimized condition was studied on the PEDOT:PSS by means of the thickness control and modification of the internal structure via addition of different organic solvents in mixture solution for thin film process.
\end{abstract}

Keywords: organic thin film solar cell, hole transfer layer, PEDOT:PSS, bulk heterojunction, $\mathrm{PCDTBT:PC}{ }_{71} \mathrm{BM}$

\section{Introduction}

Today the exhaustion of energy sources is the most critical issue for modern civilization, which is mainly due to drastic decrease of the remaining fossil fuel based energy resources by continuous and increasing utilization for the conventional electric power generation system. Moreover, environment pollution is another severe problem contributed by carbon dioxide emission from the thermoelectric power generation and radioactive wastes from the nuclear power generation. Focused on these problems of environment and being exhausted energy resources, an environment-friendly generation technology of electricity has been developed since decades, and solar-energy based solar cells have attracted much attention. Enormous amount of the solar light is emanated to the earth and it could be utilized to generate electricity without emission of harmful wastes, and therefore, the solar cell is regarded as an environment-friendly energy generation technology. Nevertheless, current supply price of the electricity via Si-based inorganic solar cell is still relatively expensive (e.g., $30 \mathrm{Yen} / \mathrm{kWh}$ in Japan) compared to that (e.g., $22 \mathrm{Yen} / \mathrm{kWh}$ in Japan) of the electricity supplied by conventional electric power company. Therefore, price cutting for consumer electricity by reduced production cost would be a critical issue for popularization of the environment-friendly electricity based on solar cell technology. Topics of almost all the solar cell researches are nowadays focused on the low-cost generation of electric power.

Recently, organic thin film solar cell (OTFSC) based on organic semiconductor materials have attracted much attention to solve the above-mentioned problems of environment-friendly and low-cost power generation. Easier and low-cost fabrication technologies distinguished from those of conventional inorganic counterparts would be a typical advantage for the OTFSCs (Pivrikas, Neugebauer, \& Sariciftci, 2011). In addition, light-weight originated from the organic material itself, flexibility, and three-dimensional layout could be other merits for OTFCs. If the OTFSCs combined with such advantages could be realized, then wearable or portable power source units could be popularized, and the place for its utilization could be much extended: although the entire amount of the solar energy emitted to the earth is huge enough, but it is strictly limited to the size of being emanated area (e.g., $1.37 \mathrm{~kW} / \mathrm{m}^{2}$ ). An accelerated supply of the solar light power generation could only be 
realized by low-price solar cell device, and various kinds of new design and technologies are now under development for the realization of those solar cell devices (Kaltenbrunner et al., 2012). One of the prime candidates might be introduction of existing printing technologies combined with flexible substrates like plastics. A low-cost production for solar cell device could be realized by means of those methodologies and therefore, series of studies reports recently drastic advances of the technologies.

Recently introduced thin film Si solar cells are discrete devices based on semiconductor functions of inorganic materials, and large number or organic materials have revealed similar semiconducting characteristics. Such organic semiconductors could be used to substitute for the inorganic counterparts for fabrication of low-cost process based thin film solar cells. Initially presented design for OTFSCs was a simple structure of p-type organic semiconductor interfaced with a metal electrode of different work function. Energy conversion performance of such devices could hardly be improved, and an appropriate solution to overcome the disadvantage has been found from the development and commercialization for other organic electronic device such as organic light emitting diodes (OLEDs). The methodology developed for OLEDs could also be adapted for organic thin film solar cells, and the p-n heterojunction type organic solar cell could reveal power conversion efficiency (PCE) of over the marginal limit of $1 \%$ (Tang, 1986). Being motivated by the success, various organic materials have been developed to drastically improve the performance of the OTFSCs. Diverse varieties of the "soccer ball-shaped" fullerene $\left(\mathrm{C}_{60}\right)$ materials have been developed for a large-scale synthesis to be used as excellent n-type organic semiconducting material. Recent success of the organic thin film solar cell has been largely contributed by introduction of bulk hetero junction structures, which is a unique concept developed exclusively for organic semiconductors (Xue, Rand, Uchida, \& Forrest, 2005). As for side of the materials, conducting materials of the conjugated polymers also have contributed to the drastic development for organic thin film solar cells ( $\mathrm{Li}$ et al., 2013). Thanks to the introduction of conjugated polymers combined with fullerene derivatives and advanced structure of bulk hetero junction, the PCEs of organic thin film solar cells have been drastically improved and these values are from $7.4 \%$ to $11 \%$ with a single device structure (Gan, Bartoli, \& Kafafi, 2013; He et al., 2012; Liang et al., 2010).

\section{Experimental Procedure}

\subsection{Materials for Fabrication of OTFSC Devices}

To study the effect of hole transfer layer of PEDOT:PSS on performance of resulting OTFSC devices, representative bulk heterojunction

Poly[9-(1-octylnonyl)-9H-carbazole-2.7-diyl]-2.5-thiophenediyl-2.1.3-benzothiadiazole-4.7-diyl-2.5-thiophenedi yl] (PCDTBT) and [6,6]-Phenyl C71 butyric acid methyl ester $\left(\mathrm{PC}_{71} \mathrm{BM}\right)$ were adopted for preparation of active layer (You et al., 2013), where PCDTBT (1-Material) is as an electron donor and $\mathrm{PC}_{71} \mathrm{BM}$ (American Dye Source, ADS71BFA) is as an electron accepter. Figure 1 and Figure 2 show molecular structure of PCDTBT and $\mathrm{PC}_{71} \mathrm{BM}$, respectively. Organic thin film solar cells are based on charge separation at the interface of electron donor (D) and electron accepter (A), where excitons generated from the light absorbed composites are incorporated to an electron transfer reaction (Opitz et al., 2010). However, the separated charge carriers are likely to recombine, which is the reason of need for an additional layer for exclusive and effective transfer of either electrons or holes to appropriate electrode. Poly(3,4-ethylenedioxythiophene):poly(styrenesulfonate) is an advantageous hole transfer layer, where poly(styrenesulfonate)(PSS) is added to an conductive polymer of Poly(3,4-ethylenedioxythiophene)(PEDOT) for doping of protons to enhance the electrical conductivity. Moreover, the PSS has high water solubility, so that it could work as both for effective dopant and dispersing agent in solvent. Consequently, molecular weight of PSS is relatively high with respect to that of the PEDOT. PEDOT:PSS has several advantages for application in organic electronic devices: (1) highly soluble in aqueous solution to be used for solution based coating; (2) insoluble in organic solvent for application to multi-layer coating; (3) highly transparent as thin-film state; (4) high electrical conductivity (Fang, Wu, Xie, Geng, \& Wang, 2011). In this research, PEDOT:PSS (Heraeus, CleviosTM PH1000) was used as a hole transfer layer for the organic thin film solar cell device. Figure 3 shows molecular structure of PEDOT:PSS.

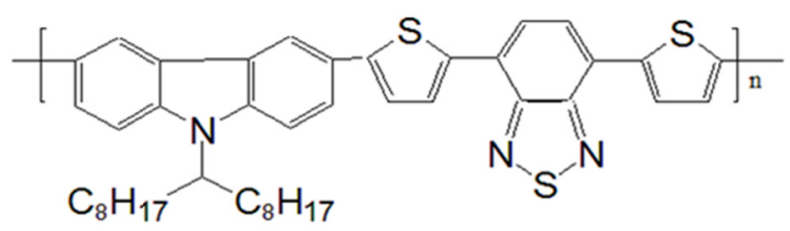

Figure 1. Molecular structure of PCDTBT 


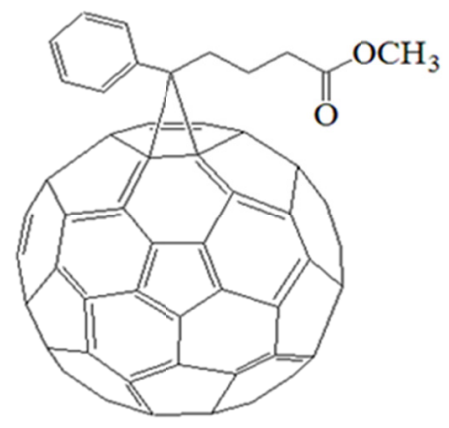

Figure 2. Molecular structure of $\mathrm{PC}_{71} \mathrm{BM}$
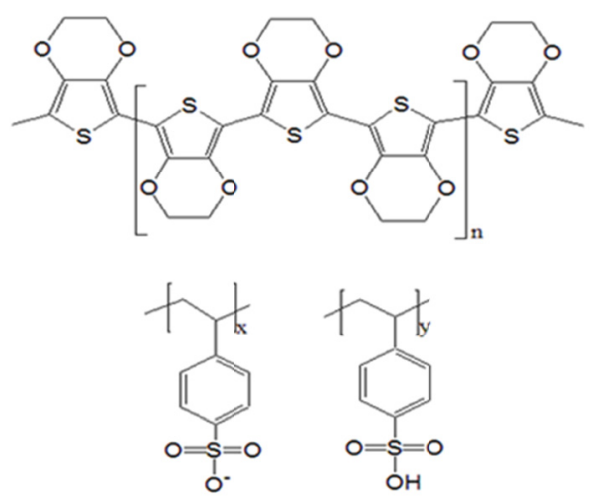

Figure 3. Molecular structure of PEDOT:PSS

\subsection{Preparation and Characterization of Organic Polymer Layers and OTFSC Devices}

Organic thin film solar cell devices were fabricated by spin-coating process: ITO as anode; PEDOT:PSS as hole transfer layer; $\mathrm{PCDTBT} / \mathrm{PC}_{71} \mathrm{BM}$ as active layer; $\mathrm{Al}$ as cathode. Figure 4 shows a schematic of the OTFSC device: (a) plan view; (b) cross-sectional view. A glass substrate coated with transparent ITO was used and cleaned ultrasonically in a sequential step with a neutral detergent, distilled water, acetone, and ethanol for 10 min, respectively. The cleaned substrates were exposed to UV in vacuum for complete drying. OTFSC devices were then fabricated either without PEDOT:PSS or PEDOT:PSS with variable thickness and different mixture solutions. Thickness of the PEDOT:PSS was varied by varying the rotation speed in the related spin-coating process for $30 \mathrm{~s}$ : (1) $3000 \mathrm{rpm}$; (2) $5000 \mathrm{rpm}$; (3) $7000 \mathrm{rpm}$. In addition, three variations of the PEDOT:PSS were also adopted for the hole transfer layer preparation: (1) pristine PEDOT:PSS; (2) PEDOT:PSS with an additive of ethylene glycol (EG; $0.05 \mathrm{ml}$ ); (3) PEDOT:PSS with an additive of dimethyl sulfoxide (DMSO; 0.05 $\mathrm{ml})$. The active layer was then prepared by spin-coating process $(1000 \mathrm{rpm}, 60 \mathrm{~s})$ with PCDTBT $(4.48 \mathrm{mg})$ and $\mathrm{PC}_{71} \mathrm{BM}(17.92 \mathrm{mg})$ dissolved in chlorobenzene of $1 \mathrm{ml}$. Finally, Al thin film as cathode was deposited by thermal evaporation process in vacuum. The resulting organic thin film solar cell devices have an effective active layer of $25 \mathrm{~mm}^{2}(5 \mathrm{~mm} \times 5 \mathrm{~mm})$. Current-voltage characteristics of the OTFSC devices were measured by employing an AC meter (Advantest R-6441) in an commercial solar simulator with an air mass $1.5 \mathrm{G}$ (AM 1.5 G) filter. Open circuit voltage (Voc), short circuit current density (Jsc), fill factor (FF), and power conversion efficiency (PCE) were obtained from the I-V characteristics for OTFSC devices. Light absorption characteristics of the organic polymer layers were analyzed by a UV/vis spectrophotometer (Shimazu, UV2450). Surface morphology was also investigated for bare ITO/glass and PEDOT:PSS (5000 rpm) coated ITO/glass. Molecular orientation of the organic polymer layers were analyzed by X-ray diffraction (Shimazu, XD-D1) technique.

\section{Results and Discussion}

\subsection{Optimization of the Thickness of PEDOT:PSS}

Effect of the thickness of PEDOT:PSS was studied on four different varieties of organic thin film solar cell devices: (1) without PEDOT:PSS; (2) with PEDOT:PSS (3000 rpm); (3) with PEDOT:PSS (5000 rpm); (4) with PEDOT:PSS (7000 rpm). Current density $\left(\mathrm{A} / \mathrm{cm}^{2}\right)$ - voltage (V) characteristics of the organic thin film solar cell devices are shown in Figure 5. 


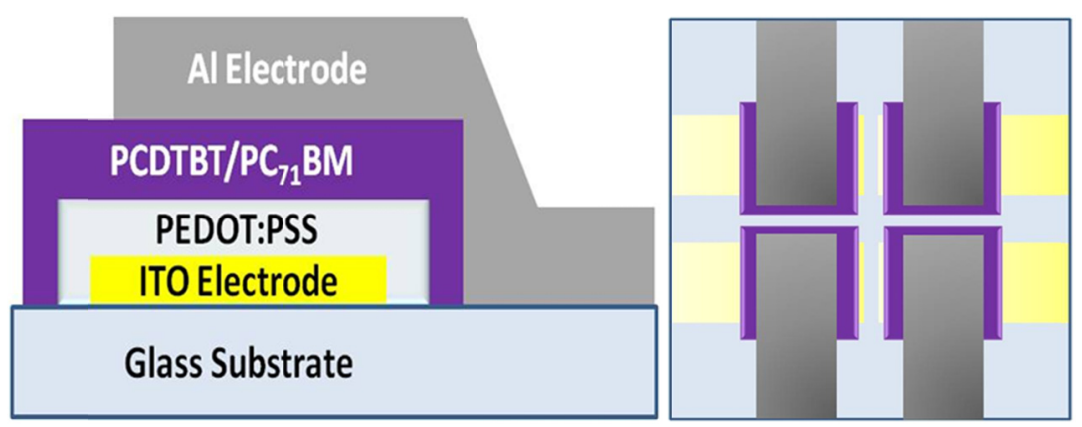

Figure 4. Schematics of OTFSC devices: (a) top view; (b) cross-sectional view

Open circuit voltage $\left(\mathrm{V}_{\mathrm{oc}}\right)$, short circuit current density $\left(\mathrm{J}_{\mathrm{sc}}\right)$, fill factor $(\mathrm{FF})$, and power conversion efficiency (PCE) of the organic thin film solar cell devices are summarized in Table 1. While the organic thin film solar cells reveal minute change of the $\mathrm{V}_{\mathrm{oc}}$ due to hole transfer layer of PEDOT:PSS, $\mathrm{J}_{\mathrm{sc}}$ of the organic thin film solar cell devices shows distinct improvement due to PEDOT:PSS. FF of the organic thin film solar cell devices is also enhanced by the effect of PEDOT:PSS. It can be thought that PEDOT:PSS could work as effective hole transfer layer, and distinctively improved PCEs of the organic thin film solar cell devices are due to the increase of $\mathrm{J}_{\mathrm{sc}}$ and $\mathrm{FF}$ contributed by the effect of PEDOT:PSS. Figure 6 shows a schematic diagram of energy levels for the organic thin film solar cell device with PEDOT:PSS as hole transfer layer. Work function of the ITO of anode is $4.7 \mathrm{eV}$ and HOMO level of the electron donor of PCDTBT is $5.5 \mathrm{eV}$. The PEDOT:PSS with a work function of $5.0 \mathrm{eV}$ could be contributed to enhanced hole transfer from the electron donor of PCDTBT to the anode of ITO, which could be resulted in improved $\mathrm{J}_{\mathrm{sc}}$ and FF of organic thin film solar cell.

Change of rotation speed at the spin coating process resulted in alteration of thickness for the resulting PEDOT:PSS layers. Thicknesses of the PEDOT:PSS layers are summarized in Table 2. Thickness of the PEDOT:PSS layer is inversely proportional to rotation speed (rpm): the higher the rpm is, the thinner is the thickness of PEDOT:PSS. Regarding the J-V characteristics (Figure 5) and performance parameters (Table 1), thickness of the PEDOT:PSS could have decisive role for characteristics of the resulting organic thin film solar cell devices (Gholamkhass \& Servati, 2013; Jeong et al., 2013; Lee, Kim, Shim, \& Park, 2009). While the PEDOT:PSS layers prepared with the rotation speed of $3000 \mathrm{rpm}(94 \mathrm{~nm})$ and $5000 \mathrm{rpm}(58 \mathrm{~nm})$ resulted in distinctive improvement of performance of organic thin film solar cell device (Seifert, Albrecht, Mietke, Koehler, \& Werner, 2003), the PEDOT:PSS layer prepared with the rotation speed of $7000 \mathrm{rpm}(28 \mathrm{~nm})$ could not be effective on the performance improvement. It can be thought that there might be a critical thickness for the hole transfer layer of PEDOT:PSS to be effective and too thinner PEDOT:PSS might not be effective for enhancement of the performance of organic thin film solar cell device (Woo, Jeong, Lyu, Han, \& Kim, 2012).

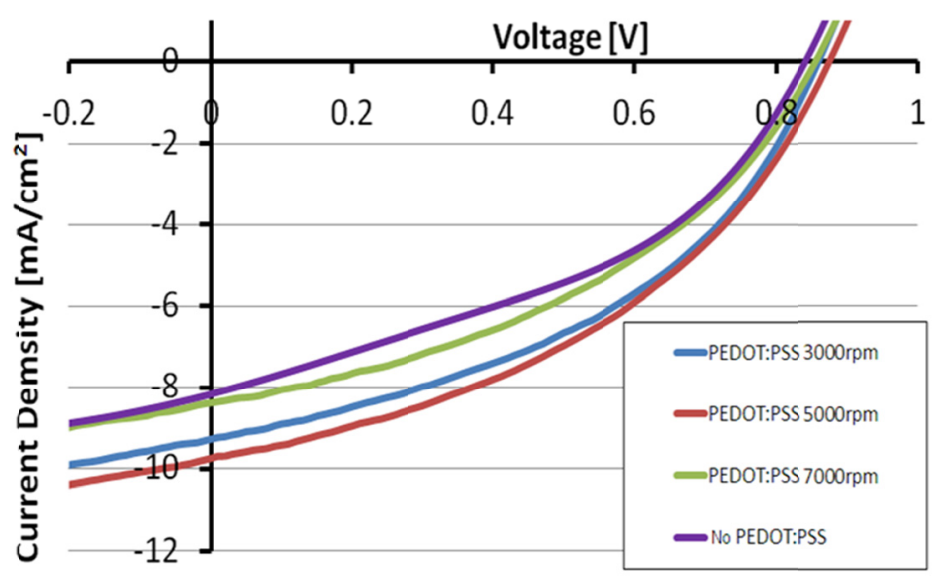

Figure 5. J-V characteristics of the organic thin film solar cell devices without PEDOT:PSS and with PEDOT:PSS of different thickness: (1) without PEDOT:PSS; (2) with PEDOT:PSS (3000 rpm); (3) PEDOT:PSS (5000 rpm); (4) with PEDOT:PSS (7000 rpm) 
Table 1. Performance parameters of the organic thin film solar cell devices without PEDOT:PSS and with PEDOT:PSS of different thickness: (1) without PEDOT:PSS; (2) with PEDOT:PSS (3000 rpm); (3) PEDOT:PSS (5000 rpm); (4) with PEDOT:PSS (7000 rpm)

\begin{tabular}{ccccc}
\hline Buffer layer & $\mathrm{V}_{\text {oc }}[\mathrm{V}]$ & $\mathrm{J}_{\mathrm{sc}}\left[\mathrm{mA} / \mathrm{cm}^{2}\right]$ & $\mathrm{FF}$ & $\mathrm{PCE}(\eta)[\%]$ \\
\hline No PEDOT:PSS & 0.84 & 8.16 & 0.41 & 2.79 \\
PEDOT:PSS (3000 rpm) & 0.86 & 9.27 & 0.43 & 3.43 \\
PEDOT:PSS (5000 rpm) & 0.87 & 9.74 & 0.42 & 3.56 \\
PEDOT:PSS (7000 rpm) & 0.85 & 8.38 & 0.41 & 2.94 \\
\hline
\end{tabular}

Table 2. Thickness of the PEDOT:PSS layers prepared by a spin coating process with three different rotation speed

\begin{tabular}{cc}
\hline Rotation speed & Thickness [nm] \\
\hline PEDOT:PSS (3000 rpm) & 94 \\
PEDOT:PSS (5000 rpm) & 58 \\
PEDOT:PSS (7000 rpm) & 28 \\
\hline
\end{tabular}

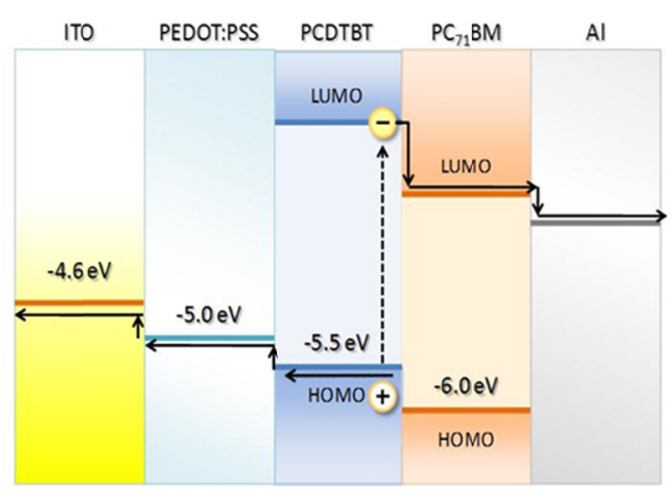

Figure 6. A schematic diagram of energy levels for the organic thin film solar cell device with PEDOT:PSS as hole transfer layer

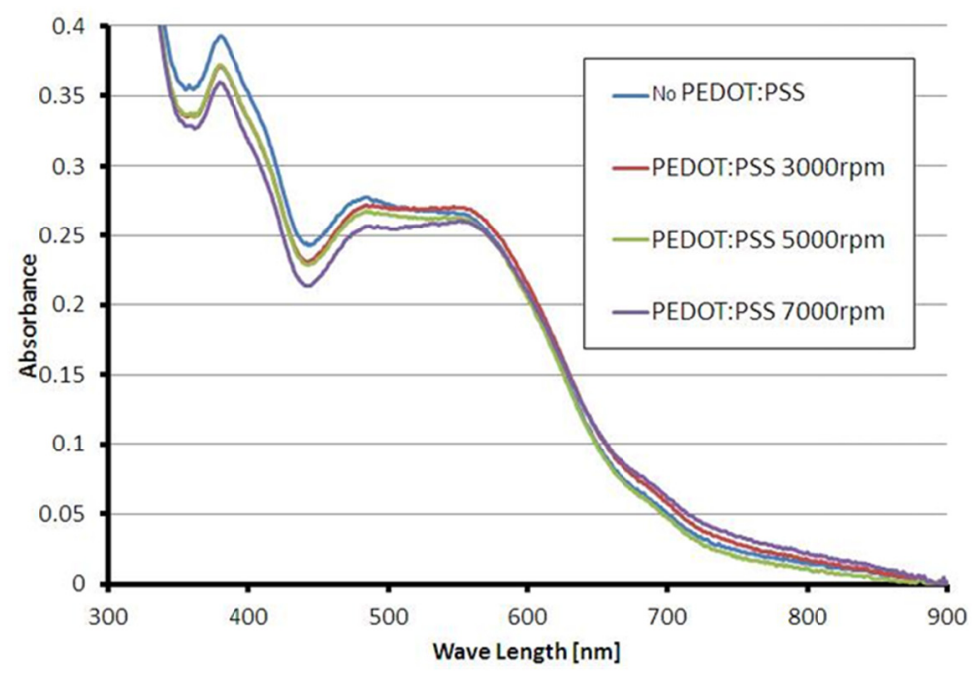

Figure 7. Light absorbance characteristics of the bare ITO (without PEDOT:PSS) and PEDOT:PSS layers on ITO: $3000 \mathrm{rpm} ; 5000 \mathrm{rpm} ; 7000 \mathrm{rpm}$ 

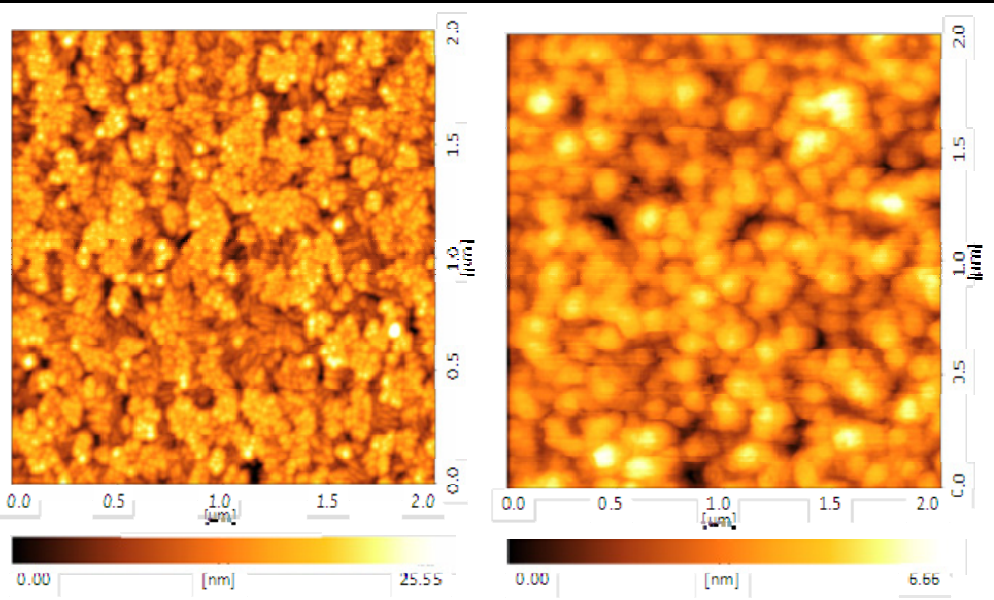

Figure 8. AFM images for ITO and PEDOT:PSS: (a) bare ITO; (b) PEDOT:PSS (5000 rpm)

Deeper insight of the thickness-effect was studied by investigation of the light absorbance characteristic (UV-vis) and surface morphology (AFM) on the three varieties of PEDOT:PSS. Figure 7 shows the light absorbance characteristic of the PEDOT:PSS layers analyzed by UV-vis spectrophotometer. Figure 8 shows an image of surface morphology of the PEDOT:PSS prepared by a rotation speed of $5000 \mathrm{rpm}$ compared with that of the ITO anode used for fabrication of organic thin film solar cell device. Difference in surface roughness was obtained from the AFM analysis for the ITO and PEDOT:PSS $(5000 \mathrm{rpm} ; 58 \mathrm{~nm})$, and root-mean-square (rms) and peak-to-valley $(\mathrm{P}-\mathrm{V})$ values are summarized in Table 3. The light absorbance characteristic curves (Figure 7) reveal that there is no distinctive variation of light-absorption-peak and light-absorption-wavelength for ITO and PEDOT:PSS layers. It can be thought that the PEDOT:PSS thin films have fairly high optical transmittance characteristic in visible wavelength range. The surface roughness data (Table 3 ) indicate that the PEDOT:PSS resulted in drastic decrease of surface roughness for both of rms and P-V values. The decreased surface roughness could be contributed to improvement of surface interface with active layers. The increased $\mathrm{J}_{\mathrm{sc}}$ of the organic thin film solar cell device (Table 1) might be originated by the enhanced charge carrier extraction due to improved surface interface (Gholamkhass \& Servati, 2013). Considering the above mentioned factors, an optimized process condition was decided for the preparation of PEDOT:PSS by spin coating technique: rotation speed of $5000 \mathrm{rpm}$; rotation time of $30 \mathrm{~s}$.

Table 3. Surface roughness of ITO and PEDOT:PSS (5000 rpm) on ITO

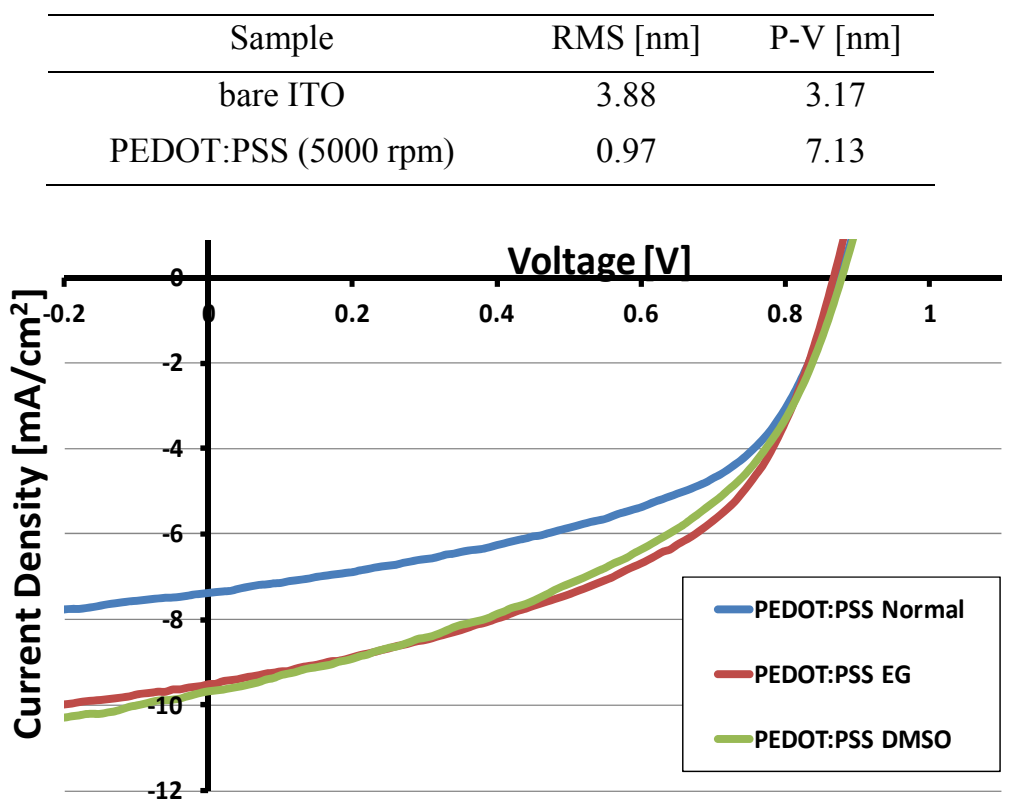

Figure 9. J-V characteristics of the organic thin film solar cell devices with three different PEDOT:PSS as hole transfer layer: (1) pristine PEDOT:PSS; (2) PEDOT:PSS added with EG; (3) PEDOT:PSS added with DMSO 


\subsection{Modification of the Property of PEDOT:PSS by Two Different Additives}

Further improvement of hole transport property of the PEDOT:PSS was tried by adding two different additives in the precursor mixture solution for preparation of the hole transfer layer of PEDOT:PSS by spin coating process (Xiao, Cui, Anderegg, Shinar, \& Shinar, 2011; Ochiai, Kumar, Santhakumar, \& Shin, 2013).
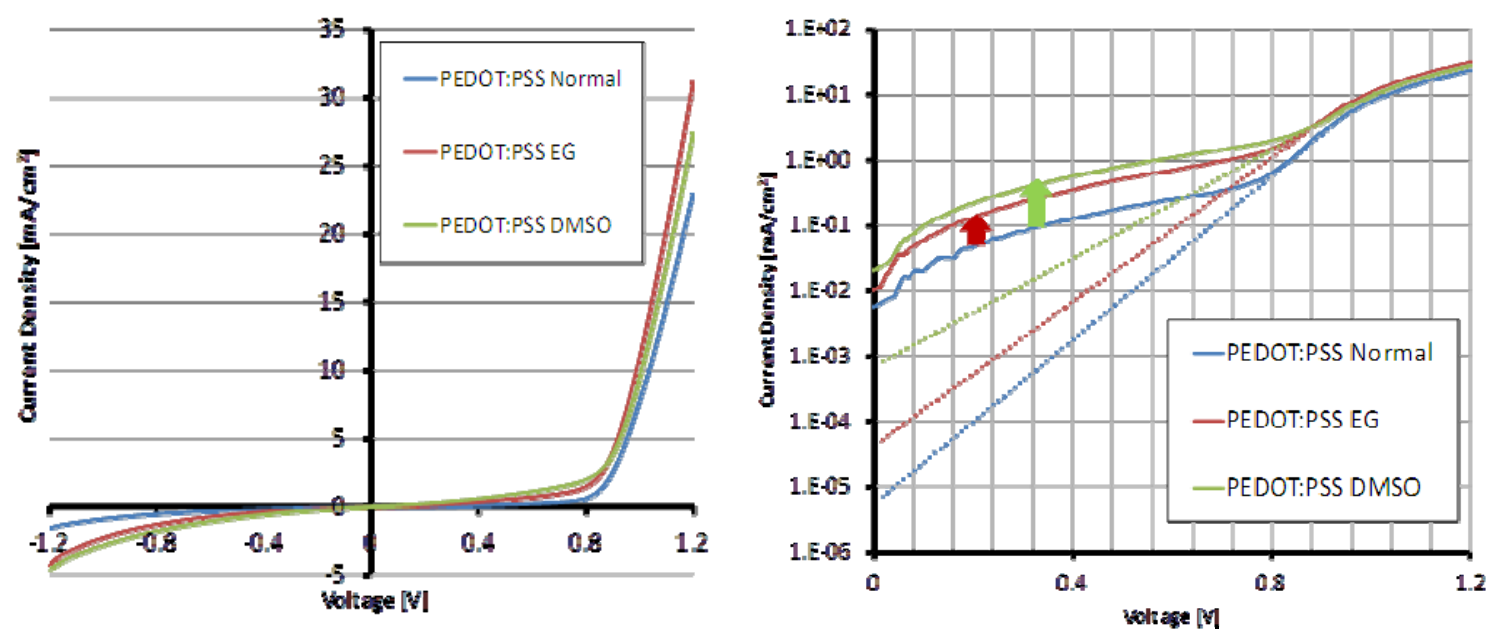

Figure 10. Dark current characteristic curves the organic thin film solar cell devices with three different PEDOT:PSS as hole transfer layer: (1) pristine PEDOT:PSS; (2) PEDOT:PSS added with EG; (3) PEDOT:PSS added with DMSO

Figure 9 shows J-V characteristic curves of three different organic thin film solar cell devices: (1) pristine PEDOT:PSS; (2) PEDOT:PSS added with EG; (3) PEDOT:PSS added with DMSO. Performance parameters obtained from the J-V characteristic curve are summarized in Table 4. The two OSC devices with PEDOT:PSS with additive reveal distinctively higher $\mathrm{J}_{\mathrm{sc}}$ than that with pristine PEDOT:PSS, while they show smaller FF values than that with pristine PEDOT:PSS. Despite the highest $J_{\mathrm{sc}}$ of $9.67 \mathrm{~mA} / \mathrm{cm}^{2}$, the OSC device with PEDOT:PSS (DMSO) reveal a drastic decrease of FF value. Consequently, the OSC device with PEDOT:PSS (DMSO) reveals rather smaller increase of PCE $(\eta=3.82 \%)$. On the other hand, the OSC device with PEDOT:PSS (EG) reveals distinctively improved PCE $(\eta=4.07 \%)$ due to combined effect of distinctively enhanced $\mathrm{J}_{\mathrm{sc}}\left(9.50 \mathrm{~mA} / \mathrm{cm}^{2}\right)$ and suppressed decrease of FF. Dark current characteristics of the three different OSC devices are shown in Figure 10: (a) rectification characteristic; (b) semi-logarithmic float. Obvious increase of dark current density can be observed for the two OSC devices with PEDOT:PSS (EG) or PEDOT:PSS (DMSO). Regarding the increase of photo-induced current and dark current, it is clear that conductivity of the PEDOT:PSS with additive has been improved, which, in turn, resulted in increase of $\mathrm{J}_{\mathrm{SC}}$. Slope of the curve at working point shown in Figure 10(b) represents rectification characteristic of the OSC device, and it could be partially related to FF at light absorption. The OSC with PEDOT:PSS (DMSO)shows distinctively mitigated slope relative to that of the OSC with pristine PEDOT:PSS. Onthe other hand, the OSC device with PEDOT:PSS (EG) reveal moderate relaxation of slope, which corresponds to the suppressed decrease of FF given in Table 4.

Table 4. Performance parameters of the organic thin film solar cell devices with three different PEDOT:PSS as hole transfer layer: (1) pristine PEDOT:PSS; (2) PEDOT:PSS added with EG; (3) PEDOT:PSS added with DMSO

\begin{tabular}{ccccc}
\hline Buffer layer & $\mathrm{V}_{\mathrm{oc}}[\mathrm{V}]$ & $\mathrm{J}_{\mathrm{sc}}\left[\mathrm{mA} / \mathrm{cm}^{2}\right]$ & $\mathrm{FF}$ & $\mathrm{PCE}(\eta)[\%]$ \\
\hline PEDOT:PSS & 0.88 & 7.39 & 0.51 & 3.31 \\
PEDOT:PSS (EG) & 0.87 & 9.50 & 0.49 & 4.07 \\
PEDOT:PSS (DMSO) & 0.88 & 9.67 & 0.45 & 3.82 \\
\hline
\end{tabular}

To clarify the effect of the additive of EG or DMSO in PEDOT:PSS on the characteristic of the resulting OSC device, surface morphology (AFM), light absorption characteristic (UV-vis spectrophotometer), and molecular 
orientation (XRD) of the PEDOT:PSS layers were investigated. Figure 11 shows images of surface morphology analyzed by AFM. Surface roughness parameters obtained from the AFM analysis are summarized in Table 5: rms [nm]; P-V [nm].
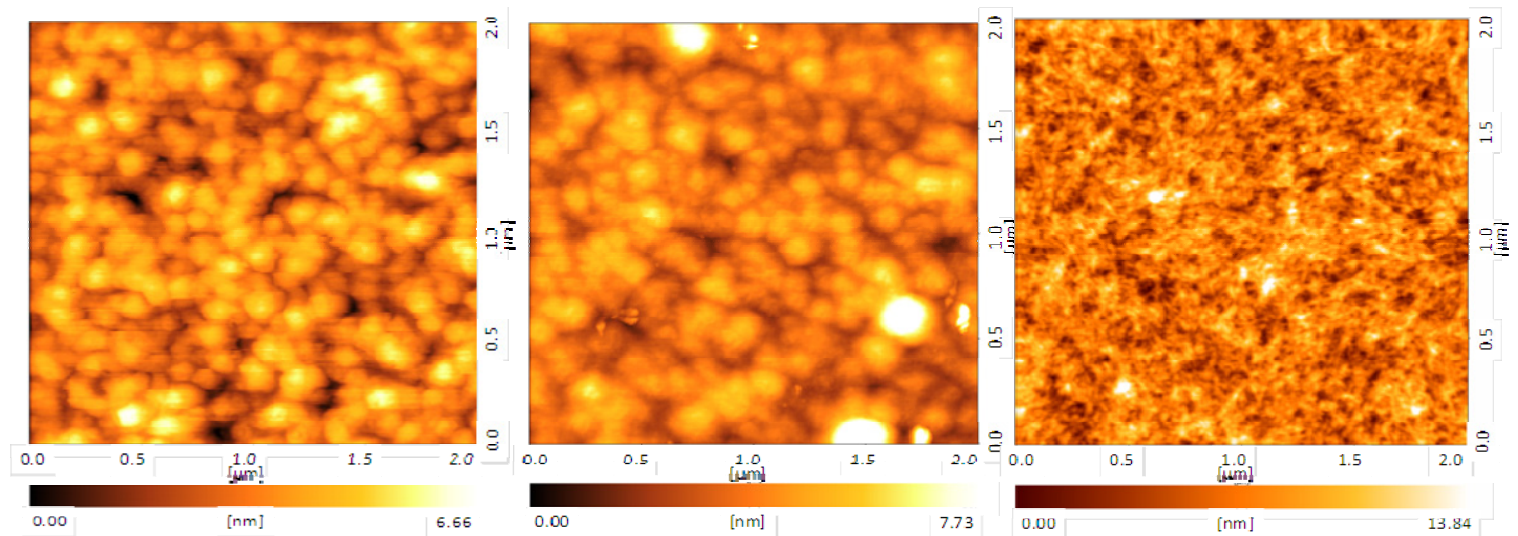

Figure 11. AFM images for three different PEDOT:PSS layers: (a) pristine PEDOT:PSS; (b) PEDOT:PSS

(DMSO); (c) PEDOT:PSS (EG)

Table 5. Surface roughness parameters obtained from AFM analysis for three different PEDOT:PSS layers: pristine PEDOT:PSS; PEDOT:PSS (EG); PEDOT:PSS (DMSO)

\begin{tabular}{ccc}
\hline Layer & RMS [nm] & P-V [nm] \\
\hline PEDOT:PSS & 0.97 & 7.13 \\
PEDOT:PSS (EG) & 1.11 & 11.89 \\
PEDOT:PSS (DMSO) & 1.59 & 18.40 \\
\hline
\end{tabular}

The pristine PEDOT:PSS reveals relatively superior surface smoothness than those of the PEDOT:PSS with additives, which is originated that PEDOT:PSS is a conducting polymer with a nature of colloid-dispersive-molecules. The PEDOT:PSS with additive of DMSO shows grains with elongated shape on the surface, which indicates that DMSO effected to promote a condensation of colloid particles. On the other hand, the PEDOT:PSS with additive of EG shows no particles on the surface, which implies that EG did not effect to hinder a condensation of particles of PEDOT:PSS but rather to be formed as needle-shapes (Nano-morphology). Although the PEDOT:PSS with additive of EG reveals clearly increased surface roughness (rms and P-V value) compared to that of the pristine PEDOT:PSS, such a change of the surface morphology might be contributed to modification of internal structure of active layers for improved power conversion efficiency: increase of FF and parallel resistance; decrease of serial resistance.

Table 6. Thickness of the three different PEDOT:PSS layers: pristine PEDOT:PSS; PEDOT:PSS (EG); PEDOT:PSS (DMSO)

\begin{tabular}{cc}
\hline Layer & Thickness [nm] \\
\hline PEDOT:PSS & 58 \\
PEDOT:PSS (EG) & 28 \\
PEDOT:PSS (DMSO) & 39 \\
\hline
\end{tabular}

Thickness of the PEDOT:PSS layers are given in Table 6. Thicknesses of the PEDOT:PSS with additives are thinner than that of the pristine PEDOT:PSS, which is possibly due to higher melting point of the organic solvent including the additives of EG $\left(\mathrm{T}_{\mathrm{m}}=189^{\circ} \mathrm{C}\right)$ or DMSO $\left(\mathrm{T}_{\mathrm{m}}=197{ }^{\circ} \mathrm{C}\right)$ : drying after spin casting might be done slowly. Although too thin layer of PEDOT:PSS could not be worked effectively as buffer layer, the PEDOT:PSS with additive of EG revealed suppressed decrease of FF (Table 4). That might be closely related to the surface morphology analyzed by AFM, so that increase of specific surface area due to additive effected to suppress the 
decrease of FF. To examine molecular orientation of the PEDOT:PSS layers, XRD profiles were investigated. Figure 12 shows XRD profiles of pristine PEDOT:PSS layers prepared by spin-coating and drop-casting process. Any broad diffraction peaks cannot be observed for the spin-coated PEDOT:PSS (rotation speed of $5000 \mathrm{rpm}$; rotation time of $30 \mathrm{~s}$ ) at the measurement condition: measurement range $0-30^{\circ}$; measurement resolution $3^{\circ}$. The broad diffraction peak observed at $25^{\circ}$ could be related to condensation of PEDOT:PSS due to slow diffusion of droplet during the drop casting process.

Therefore, PEDOT:PSS layers prepared by drop casting technique ( $0.2 \mathrm{ml}$ of PEDOT:PSS solution) were used to examine the crystallinity. Figure 13 shows XRD spectra (out-of-plane) for the three PEDOT:PSS layers prepared by drop casting process: (1) pristine PEDOT:PSS; (2) PEDOT:PSS (EG); (3) PEDOT:PSS (DMSO). The PEDO' $: P S S$ (DMSO) reveals two broad peaks at $2 \theta=20^{\circ}$ and $2 \theta=25^{\circ}$ (Cho et al., 2005), which are closed related to the grains observed at the surface from AFM analysis. The PEDOT:PSS (EG) shows another broad peak at $2 \theta=9^{\circ}$, which is not observed at the PEDOT:PSS (DMSO). Regarding the Bragg's condition ( $2 \mathrm{~d} \sin \theta=$ $\mathrm{n} \lambda$ ), lattice distance for that case could be approximately $4.9 \AA$ A. The new diffraction peak might be appeared due to enlarged rod-shape grain by the effect of EG. Finally, light absorbance characteristics were investigated by UV-vis spectrophotometer for active layer (PCDTBT:PC ${ }_{71} \mathrm{BM}$ ) prepared on the three PEDOT:PSS layers, and Figure 14 shows the result. Independent on the underlying PEDOT:PSS layer, all the composite layers reveal similar light absorption characteristics, which indicates that light absorption characteristic of the PEDOT:PSS is not affected by its crystallinity and the additives have no influence on the light absorption property of active layers. Consequently, it can be thought that the obtained increased PCE was not originated by the active layer itself but the buffer layer.

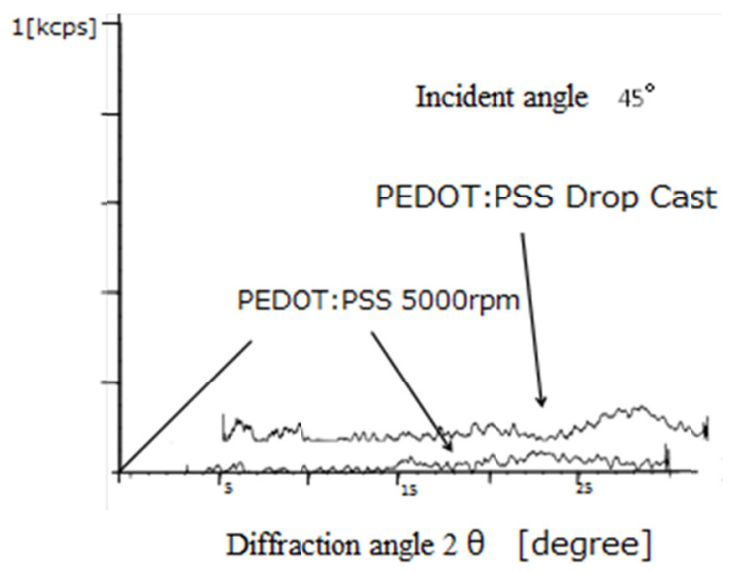

Figure 12. XRD spectra for pristine PEDOT:PSS layers prepared by spin coating and drop casting process

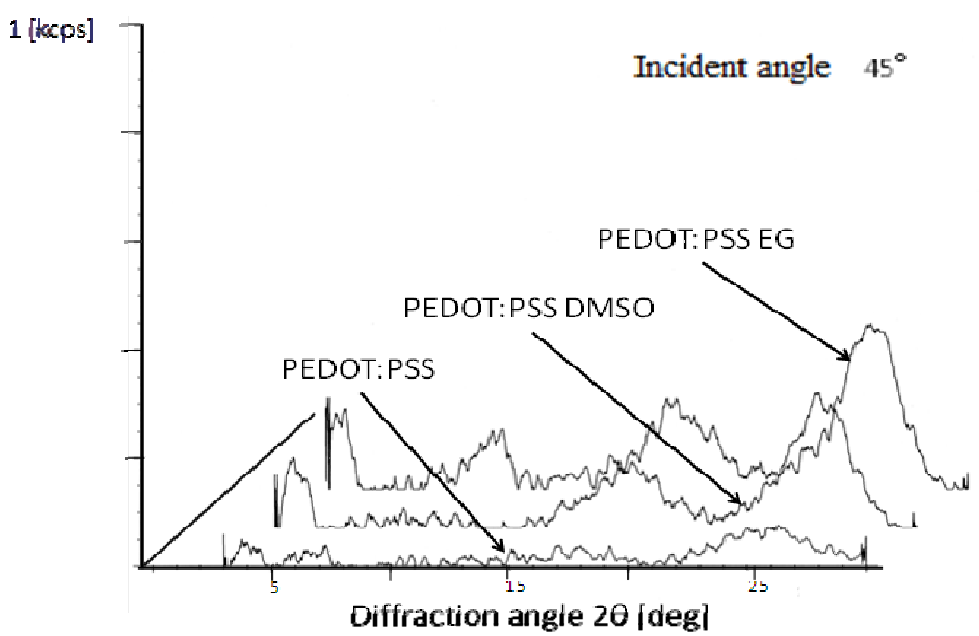

Figure 13. XRD spectra (out-of-plane) for three different PEDOT:PSS layers prepared by drop casting process 


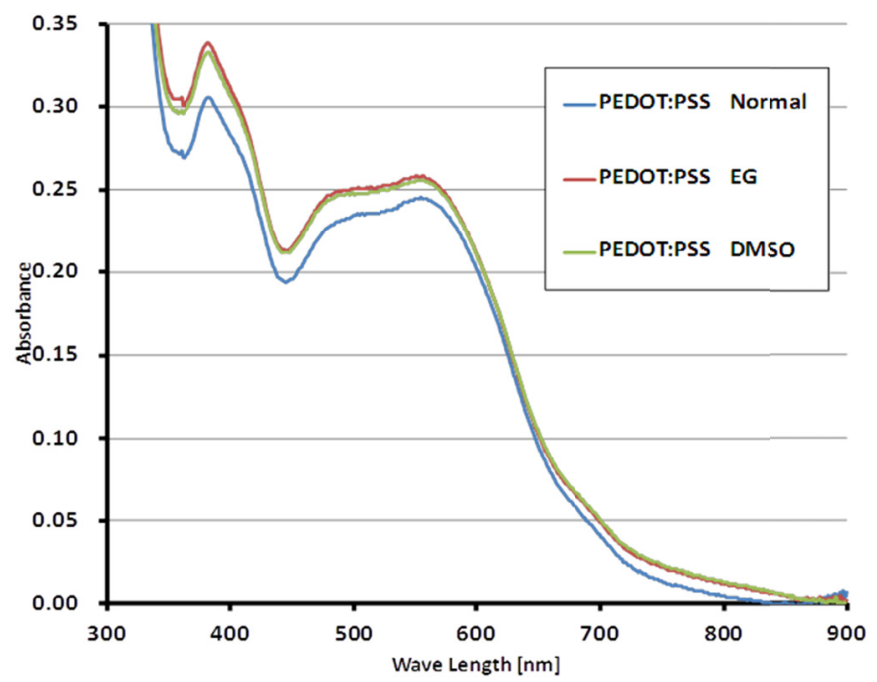

Figure 14. Light absorption characteristics of PCDTBT:PC ${ }_{71} \mathrm{BM}$ layers prepared on pristine PEDOT:PSS, PEDOT:PSS (EG), and PEDOT:PSS (DMSO)

\section{Summary and Conclusions}

In this report, a hole transfer layer of PEDOT:PSS was investigated aiming for performance improvement of OTFSC device. Representative bulk heterojunction structure of PCDTBT:PC ${ }_{71} \mathrm{BM}$ was prepared by spin coating technique to study the effect of the PEDOT:PSS. Varieties of PEDOT:PSS layers were prepared by spin coating technique, where thickness of the PEDOT:PSS layers were varied and different precursor solution mixtures of the PEDOT:PSS were used with an additive of EG or DMSO. Effect of thickness and additives were examined for varieties of PEDOT:PSS layers and/or in combination of active layers of PCDTBT:PC ${ }_{71} \mathrm{BM}$ : light absorption characteristics (UV-vis spectroscopy); surface morphology (AFM); molecular orientation (XRD). Performance parameters of the resulting OTFSC devices were investigated in relation with the characteristics of PEDOT:PSS layers. Thickness of the PEDOT:PSS could have decisive role for characteristics of the resulting OTFSC devices. The PEDOT:PSS with thickness from 58 to $94 \mathrm{~nm}$ was appropriate for performance improvement of OTFSC device by effective role of hole transfer layer, whereas thinner PEDOT:PSS of thickness of $28 \mathrm{~nm}$ was not effective. PEDOT:PSS layers prepared with additives of EG or DMSO were attributed to improve the performance of OTFSC devices, which was achieved mainly by conspicuous increase of short circuit current density, although fill factor of the OTFSC devices with PEDOT:PSS with additives decreased compared to that of the OTFSC device with pristine PEDOT:PSS. Rectification characteristic at dark current condition revealed that the increase of short circuit current density was resulted from an improvement of conductivity of the PEDOT:PSS. Especially, the PEDOT:PSS (EG) layer of $28 \mathrm{~nm}$ revealed more effective role as a hole transfer layer for OTFSC device to improve the PCE $(\eta=4.07)$, which was achieved by combined effect of increased short circuit current density $\left(\mathrm{J}_{\mathrm{sc}}=9.50 \mathrm{~mA} / \mathrm{cm}^{2}\right)$ and suppressed decrease of fill factor $(\mathrm{FF}=0.49)$. The positive effect of the PEDOT:PSS to improve the performance of the OTFSC device was found to be possibly related to surface morphology after AFM analysis.

\section{Acknowledgements}

This research was partly supported by the MEXT-Supported Program for the Strategic Research Foundation at Private Universities (2010-2014), and the joint research between Aichi Institute of Technology and NDS.

\section{References}

Cho, S., \& Lee, K. (2005). Heat-Treatment-Induced enhancement in the optical spectra of poly(3,4-ethylenedioxythiophene)/poly(stylenesulfonate) films. Journal of the Korean Physical Society, 46(4), 973-976. http://dx.doi.org/10.3938/jkps.46.973

Fang, G., Wu, S., Xie, Z., Geng, Y., \& Wang, L. (2011). Enhanced performance for polymer solar cells by using surfactant-modified PEDOT:PSS as the anode buffer layer. Macromolecular Chemistry and Physics, 212(17), 1846-1851. http://dx.doi.org/10.1002/macp.201100102 
Gan, Q., Bartoli, F. J., \& Kafafi, Z. H. (2013) Organic photovoltaics: Plasmonic-Enhanced organic photovoltaics: breaking the $10 \%$ efficiency barrier. Advanced Materials, 25(17), 2377. http://dx.doi.org/10.1002/adma.201370107

Gholamkhass, B., \& Servati, P. (2013). Solvent-Vapor induced morphology reconstruction for efficient PCDTBT based polymer solar cells. Organic Electronics, 14(9), 2278-2283. http://dx.doi.org/10.1016/j.orgel.2013.05.014

He, Z., Zhong, C., Su, S., Xu, M., Wu, H., \& Cao, Y. (2012). Enhanced power-conversion efficiency in polymer solar cells using an inverted device structure. Nature Photonics, 6(9), 591-595. http://dx.doi.org/10.1038/nphoton.2012.190

Jeong, J., Woob, S., Park, S., Kim, H., Lee, S. W., \& Kim, Y. (2013). Wide range thickness effect of hole-collecting buffer layers for polymer:fullerene solar cells. Organic Electronics, 14(11), 2889-2895. http://dx.doi.org/10.1016/j.orgel.2013.08.008

Kaltenbrunner, M., White, M. S., Głowacki, E. D., Sekitani, T., Someya, T., Sariciftci, N. S., \& Bauer, S. (2012). Ultrathin and lightweight organic solar cells with high flexibility. Nature Communications, 3, 770-777. http://dx.doi.org/10.1038/ncomms1772

Li, K., Li, Z., Feng, K., Xu, X., Wang, L., \& Peng, Q. (2013). Development of large band-gap conjugated copolymers for efficient regular single and tandem organic solar cells. J. Am. Chem. Soc., 135, 13549-13557. http://dx.doi.org/10.1021/ja406220a

Lee, S.-H., Kim, J.-H., Shim, T.-H., \& Park, J.-G. (2009). Effect of interface thickness on power conversion efficiency of polymer photovoltaic cells. Electron. Mater. Lett., 5(1), 47-50. http://dx.doi.org/10.3365/eml.2009.03.047

Liang, Y., Xu, Z., Xia, J., Tsai, S.-T., Wu, Y., Li, G., ... Yu, L. (2010). For the bright future-bulk heterojunction polymer solar cells with power conversion efficiency of 7.4\%. Adv. Mater., 22(20), E135-E138. http://dx.doi.org/10.1002/adma.200903528

Ochiai, S., Kumar, P., Santhakumar, K., \& Shin, P. (2013). Examining the effect of additives and thicknesses of hole transport layer for efficient organic solar cell devices. Electronic Materials Letters, 9(4), 399-403. http://dx.doi.org/10.1007/s13391-013-0013-5

Opitz, A., Wagner, J., Brutting, W., Salzmann, I., Koch, N., Manara, J., ... Schreiber, F. (2010). Charge separation at molecular donor-acceptor interfaces: correlation between morphology and solar cell performance. IEEE Journal of Selected Topics in Quantum Electronics, 16(6), 1707-1717. http://dx.doi.org/10.1109/JSTQE.2010.2048096

Pivrikas, A., Neugebauer, H., \& Sariciftci N. S. (2011). Influence of processing additives to nano-morphology and efficiency of bulk-heterojunction solar cells: A comparative review. Solar Energy, 85(6), 1226-1237. http://dx.doi.org/10.1016/j.solener.2010.10.012

Seifert, W., Albrecht, H., Mietke, S., Koehler, T., \& Werner, M. (2003). Processing and electrical characterization in intrinsic conducting polymers for electronic and MEMS applications. Proc. SPIE, 5045, 183. http://dx.doi.org/10.1117/12.484280

Tang, C. W. (1986). Two-Layer organic photovoltaic cell. Appl. Phys. Lett., 48, 183-185. http://dx.doi.org/10.1063/1.96937

Woo, S., Jeong, J. H., Lyu, H. K., Han, Y. S., \& Kim, Y. (2012). In situ-prepared composite materials of PEDOT:PSS buffer layer-metal nanoparticles and their application to organic solar cells. Nanoscale Research Letters, 7, 641-646. http://dx.doi.org/10.1186/1556-276X-7-641

Xiao, T., Cui, W., Anderegg, J., Shinar, J., \& Shinar, R. (2011). Simple routes for improving polythiophene:fullerene-based organic solar cells. Organic Electronics, 12, 257-262. http://dx.doi.org/10.1016/j.orgel.2010.11.008

Xue, J., Rand, B. P., Uchida, S., \& Forrest, S. R. (2005). Mixed donor-acceptor molecular heterojunctions for photovoltaic applications. II. Device performance. J. Appl. Phys., 98, 124903-9. http://dx.doi.org/10.1063/1.2142073

You, J., Chen, C.-C., Hong, Z., Yoshimura, K., Ohya, K., Xu, R., ... Yang, Y. (2013). 10.2\% Power conversion efficiency polymer tandem solar cells consisting of two identical sub-cells. Advanced Materials, 25(29), 3973-3978. http://dx.doi.org/10.1002/adma.201300964 


\section{Copyrights}

Copyright for this article is retained by the author(s), with first publication rights granted to the journal.

This is an open-access article distributed under the terms and conditions of the Creative Commons Attribution license (http://creativecommons.org/licenses/by/3.0/). 